\title{
Partager des savoir-faire pédagogiques : retours sur l'expérience éditoriale des Feuilles de géographie
}

Équipe éditoriale des Feuilles de Géographie, Sylvestre Duroudier, Leïla Frouillou, Brenda Le Bigot, Annaig Oiry, Élise Olmedo, Étienne Toureille et Camille Vergnaud

\section{(QpenEdition}

\section{Journals}

Édition électronique

URL : http://journals.openedition.org/cdg/1146

DOI : $10.4000 /$ cdg. 1146

ISSN : 2107-7266

\section{Éditeur}

UMR 245 - CESSMA

\section{Référence électronique}

Équipe éditoriale des Feuilles de Géographie, Sylvestre Duroudier, Leïla Frouillou, Brenda Le Bigot, Annaig Oiry, Élise Olmedo, Étienne Toureille et Camille Vergnaud, «Partager des savoir-faire pédagogiques : retours sur l'expérience éditoriale des Feuilles de géographie », Carnets de géographes [En ligne], 10 | 2017, mis en ligne le 01 octobre 2017, consulté le 04 mai 2019. URL : http:// journals.openedition.org/cdg/1146; DOI : 10.4000/cdg.1146

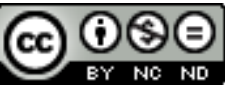

La revue Carnets de géographes est mise à disposition selon les termes de la Licence Creative Commons Attribution - Pas d'Utilisation Commerciale - Pas de Modification 4.0 International. 


\title{
Partager des savoir-faire pédagogiques. Retours sur l'expérience éditoriale des Feuilles de géographie
}

\author{
Équipe éditoriale des Feuilles de Géographie \\ (Sylvestre Duroudier, Leïla Frouillou, Brenda Le Bigot, Annaig Oiry, Élise Olmedo, \\ Étienne Toureille, Camille Vergnaud)
}

Née au début des années 1990, l'associationFeuilles de Géo, nommée familièrement Feuilles de choux, était une coopérative de partage de cours à destination des enseignants en géographiedans le supérieur. Aujourd'hui méconnue des plus jeunes générations d'enseignants-chercheurs, le projet étaitinédit àl'époque : parler pédagogie en géographie à I'Université, quand"montrer ce qu'on faisait en cours, c'était quasiment obscène » $(\mathrm{CG})^{1}$.Selon les fondateurs, la revue eut pourtant un certain succès, jusqu'à être reconnue dans des comités de sélection pour les recrutements universitaires.Son objectif était notamment de créer des supports pour s'adapter aux nouveaux formats de cours comme les travaux dirigés(TD), en proposantpar exemple des progressions d'exercices sur le modèle de manuelsanglo-américains ${ }^{2}$.

À partirdes années 2000, confronté au passage au numérique et à l'émergence d'autres revues (comme celle deGéoconfluences par exemple), le modèle des Feuilles de Géos'essouffle progressivement. Plus récemment, un passage de témoins'est opéré vers quelques doctorants qui ont constitué une nouvelle équipe éditoriale ${ }^{3}$. Nous avons saisi l'appel à communications des Carnets de géographes pour interroger la place des Feuilles de géo, en tant que revue spécifiquement dédiée aux questions pédagogiques en géographie à l'université. À travers une perspective historique, nous souhaitons questionner le fonctionnement, les objectifs et le rôlede cette revue depuis les années 1990.

\footnotetext{
${ }^{1}$ Les initiales renvoient aux différents enquêtés : Myriam Baron (MB), Sandrine Berroir (SB), Claude Grasland (CG), Renaud Le Goix (RLG), Antonine Ribardière (RB) et Georgette Zrinscak (GZ).

${ }^{2}$ Par exemple l'ouvrage de P.J. Taylor (1977), Quantitative Methods in Geography, Houghton Mifflin, cité par CG comme référence à l'époque.

${ }^{3}$ Le passage de témoin s'est fait à la fin 2012, lorsque quelques membres de l'équipe initiale ont donné carte blanche à certains d'entre nous pour reprendre la revue. Ainsi, une petite équipe de doctorants franciliens (inscrits dans les universités de Paris 1, Paris 7 et Paris 10) s'est constituée en comité éditorial et a développé un nouveau site internet pour renouveler les Feuilles de géo.
} 
Nous avons donc sollicitéles anciens porteurs de la revue. Plusieurs générations d'enseignants chercheurs, certains membres fondateurs (Myriam Baron, Claude Grasland, GeorgetteZrinscak) etparticipants successifs (Sandrine Berroir,Renaud Le Goix, Antonine Ribardière), ont été rencontrés.Ces entrevues ont eu lieuenfévrier 2016, lors de trois entretiens collectifs, etont fait dialoguer un binôme d'anciens membres et deux personnes de la nouvelle équipe. Ces échangesautour des origines, des évolutions,des limites et perspectives du projet, nous permettent ici de proposer des éléments de discussion autour de la place de la pédagogie à l'Université et du sensactuel d'une revue d'échanges de cours telle que Feuilles de géographie.

\section{Genèse : « Avant les Feuilles de géographie était le néant... " (GZ)}

\section{La solitude des jeunes enseignants}

Après une période de massification de l'enseignement supérieur durant les années 1970-80, I'Université française connait dans les années 1990 une "augmentation des personnels enseignants [...] avec le début des monitorats, des allocs, des $\operatorname{ATER}^{4}[. .$.$] . II y a eu une masse$ de jeunes qui sont arrivés en même temps. Alors qu'avant, c'était beaucoup plus progressif » (GZ).Cette nouvelle cohorte se trouve rapidement confrontée à la nécessité de faire face à la préparation des cours: " on se sentait démuni [...] c'est parti de cette idée de mettre en commun les choses, de pouvoir échanger, [...] à la fois sur le contenu et sur les pratiques » (SB).

En plus de l'impératif de service, ces nouveaux arrivants à l'Université, se trouvent propulsés dans les salles de cours sans y être préparés, ni même formés: "Y'avait pas d'effort de réflexion sur la pédagogie dans l'enseignement à l'Université. Y'avait une espèce d'approche essentialiste, dès l'instant où tu arrivais à l'Université, tu savais faire un cours. La question se posait même pas de: à qui tu t'adressais, comment tu transmettais, pourquoi tu transmettais plutôt à ce moment-là ou plutôt à tel autre moment, y'avait RIEN.[...] Au début c'était de la fausse interactivité [...] il m'a fallu cinq ans pour apprendre à écouter, à poser une question, écouter les réponses et réagir sur les réponses, et comprendre qu'en TD tu étais tout le temps sans filet. Tu savais pas à quel moment tu allais finir un truc, où tu allais arriver $»(\mathrm{GZ})$.

Savoir faire un cours, ça s'apprend: G.Z raconte son expérience de cours raté à des historiens de deuxième année

"Moi, j'avais pas l'impression de savoir-faire un cours à l'Université. Donc j'avais un premier TD qui était des historiens de deuxième année, qui étaient obligés de faire de la géographie, trop dur. Obligés. Je savais pas ce qu'ils valaient, ce qu'ils avaient dans le ventre, ce qu'ils connaissaient. Je leur ai sorti un cours d'agreg., forcément. Au bout de deux heures j'ai dit : oups ! y'a quelque chose qui va pas. C'était en 1990. Et au bout d'un an et demi, deux ans de discussions, les membres fondateurs, on était 13 [...] on s'est dit : il faut monter un truc »

\footnotetext{
${ }^{4}$ II s'agit de différents statuts permettant de donner des cours à l'Université, qu'il s'agisse de contrats, de missions ou d'autres financements.
} 
En plus du constat de l'absence de formation, s'ajoutait pour certains le sentiment de ne pas être aidés par leurs aînés : Je me souviens, en arrivant, on avait aussi le sentiment que les gens, les enseignants en place, ne nous donnaient pas, ne diffusaient pas les cours. Je me souviens très bien, des profs avec qui je faisais le TD, pour savoir ce qui se passait en cours, je revois le prof complètement étonné de me voir assise dans l'amphi, de se demander pourquoi je venais en cours. II me prenait pour une incompétente. On n'arrivait pas à savoir ce qui se passait »(SB).

\section{Quand l'unionfait la force : « le bouillonnement des années 1990 » (MB)}

Pour faire face à ces difficultés et à ce sentiment de solitude partagés, ces doctorants prennent rapidement l'habitude de travailler ensemble : "Oui, on se disait qu'on était seul, mais on n'était pas seul [...]. On était tout un groupe de doctorants, on se filait énormément nos trucs [...].Mais par contre on se disait, mon dieu, ceux qui sont vraiment tout seuls, ça doit être terrible " (AR).Cette reconnaissance de l'hétérogénéité des situations de désarroi pédagogique fonde la volonté de créer une revue qui s'étende à d'autres établissements et qui puisse être appropriée par d'autres enseignants.D'autre part, cette prise de conscience est aussi liée à des conditions de travail similaires: "On était le résultat de l'inflation du nombre de contrats d'allocataires moniteurs et d'ATER. La résultante c'est qu'effectivement, on a eu ces statuts de trois ans, puis un an, puis un an, puis un an. Moi, j'ai été ATER deux ans, j'ai fait trois fois mes petits dossiers, etc. [...]Nous étions dans les mêmes corps, dans les mêmes statuts, avec une solidarité fonctionnelle... " (RLG).

Cette situation renforce un contexte favorable à l'émergence d'un projet collectif : Dans cette genèse, et dans cette naissance de Feuille de géo, faut voir que... Ça, ça bougeait de partout c'est-à-dire... Notre statut il était complètement revalorisé par rapport à ce qu'il avait été pendant les périodes précédentes, on était quand même au tout début de la grosse vague de massification ${ }^{5}$, et donc on était face effectivement à un public étudiant qui changeait et nous on s'en rendait pas nécessairement compte puisque de toute façon on n'avait pas suffisamment de recul dans des promos. Et donc bah, il y avait une espèce d'adaptation qui a été immédiate. On a été obligé de faire sans nécessairement regarder très loin dans le rétroviseur. Donc il y avait une espèce de bouillonnement qui était, qui était propre à cette période-là, et qui explique en partie que... Qu'on se soit engouffrés là-dedans » (MB).

\section{J-0 ? Les marches de l'Institut de Géographie de Paris : colère etvinoverde.}

CG : c'était une rencontre entre deux très grands géographes[rires], par la taille je parle. Donc on s'est retrouvé avec Louis Marrou, 2 mètres 04,[...] sur le parvis de l'institut de géographie, sortant lui de l'assemblée des géographes de Paris IV, et moi de celle de Paris-I, désespérés [rires].[...]On était tous les deux, je ne sais pas, l'équivalent d'ATER, à Paris-l et

\footnotetext{
${ }^{5}$ II est question ici de massification du corps enseignant et non pas du corps estudiantin, qui connut une période d'augmentation plus précoce dès la fin de la Seconde Guerre mondiale avec une croissance très forte du nombre d'étudiants dans les années 1960.
} 
Paris $I V$, et on parlait d'un changement de maquette. Et on sortait d'une assemblée où nos collègues voulaient refaire les mêmes choses pour quatre ans, on trouvait ça totalement désespérant. Donc voilà : on s'est dit que ce n'était pas possible, qu'il fallait faire quelque chose. Et on est donc allé chercher les copains qu'on avait autour, donc on s'est retrouvé rapidement sur des Paris-I, Paris-IV, Paris-VII.[...]Enfin voilà, pour l'anecdote, effectivement, $c^{\prime}$ est né d'un coup de colèrecontre $P 1$ et $P 4$, qui voulait chacun respectivement reproduire à l'identique.......]

$\mathrm{MB}$ : le repas fondateur, au cinquième étage du 13 de la rue du Four[rires]avec force vinoverde et Saint-Emilion [rires],c'est comme ça que le projet a pris forme, avec une inspiration supplémentaire, qui était le fait de structurer davantage, et de faire connaître davantage les TD qui ne devaient pas être seulement des faire-valoir des cours magistraux.

Ces échanges de supports pédagogiques s'inscrivent dans un contexte plus général de réflexion sur l'enseignement en $\mathrm{DEUG}^{6}$ :

" MB : Il y avait une vraie discussion autour du fait qu'à l'époque la formation au DEUG elle était quand même très dévalorisée par rapport aux classes prépas, et donc il y avait des discussions dans des groupes de travails[...]qui voulaient aussi beaucoup pousser la formation en DEUG et en licence à l'université, pour montrer qu'on était capable à la fois de faire de la géographie qui tenait la route, et qui était aussi exigeante que la formation classes préparatoires. Et puis enfin l'idée qu'on évoluait, nous, dans des vraies équipes pédagogiques, dans lesquels on avait la chance de récupérer des matériaux, qui nous servaient, qui nous servaient pas, qu'on critiquait, ou qu'on réutilisait, etc. [...]

CG : on avait la chance à une échelle très réduite qui était l'échelle d'un ou deux enseignements, d'avoir des coopératives, ou en gros ceux qui rentraient bénéficiaient du capital accumulé, mais devaient l'enrichir. Donc l'idée a été de passer à une échelle un petit peu différente, qui était inter-établissements ».

\section{Le développement des Feuilles: des marches de l'Institut de Géographie à un réseau d'interconnaissance interuniversitaire}

LesFeuilles de Géographie se sont développées sur une base géographique très localisée : les doctorants allocataires moniteurs à l'Institut de Géographie, pour la plupart rattachés au laboratoireGéographie-cités. Le cercle de départ ("les treize ») était ainsi restreint à un groupe d'étudiants de trois universités de Paris (Paris 1, 4, et 7) et de l'École Normale Supérieure LSH, alors localisée à Fontenay-aux-Roses : Enfin on faisait avec des gens qui étaient intéressés et c'est vrai que là je ne nous revois pas en train de nous dire qu'il faut aller chercher des gens à l'extérieur, on essaye déjà de voir ensemble qu'est-ce qu'on peut faire [...] oui, on avait quelque chosede commun ensemble, et puis il faut remettre ça dans le contexte de ces années-là, par rapport à ce qu'on faisait à cette époque avec l'équipe P.A.R.I.S. ${ }^{7}$... Il y a aussi un contexte[...]. On n'avait pas du tout envie de s'enfermer mais voilà, on faisait avec les gens qui étaient là, qu'on voyait, avec qui on avait des préoccupations

\footnotetext{
${ }^{6}$ Diplôme d'Etudes Universitaires Générales : diplôme de premier cycle correspondant à la Licence 2.

${ }^{7}$ Pour l'Avancement des Recherches sur I'Interaction Spatiale : I'une des équipes de l'UMR Géographie-cités.
} 
communes. Et puis on le faisait aussi avec l'idée que ça puisse intéresser d'autres gens, que ça puisse se diffuser.[...] C'est des gens qui se sont mis ensemble, qui avaient une certaine proximité effectivement, des positionnements dans la géographie de l'époque, voilà. " (SB).Le groupe des treize se retrouve sur les marches de l'Institut, mais rapidement tente d'essaimer au-delà de l' " effet Institut [et du] fort déterminisme spatial géographique. C'était l'effet centralité, Paris 1, Paris 4, Institut. Et Paris 7 avec le noyau où il y avait le plus d'enseignants chercheurs. [...]Y'a eu un moment : quand on a commencé à faire France entière... " (GZ).

$\mathrm{Au}$ fil des générations d'adhérents, les Feuilles de Géographies'étendent au-delà des universitésde Paris, même si le bureau reste parisiano-centré. Le développement rapide de l'association et de la revue sont significatifs des besoins des chargés de cours de l'époque :

" $R L G$ : il y avait un grand besoin.

GZ: chez les jeunes, il y a eu tout de suite une centaine de personnes dans I'assoc'.

RLG : Et le nombre de publications a tout de suite été très fort. "

Le développement de la revue se fait surtout grâce au bouche-à-oreille,au réseau d'interconnaissance, et à travers quelques manifestations importantes, comme leFestival international de Saint-Dié-des-Vosges, lancé en 1990.

\section{L'enseignement "parent pauvre » ou le désir de "rénover l'enseignement " à l'Université(CG)}

Dès le lancement du projet, les initiateurs de la revue affirment des objectifs relativement ambitieux compte tenu du chantier qui s'offre à eux. Nos interlocuteurs disent avoir toujours manifesté la volonté de répondre à des problèmes concrets et de chercher à se dégager de toute chapelle ou tutelle théorique : "on avait plusieurs choses qu'on voulait faire mais on voulait absolument rénover l'enseignement. On trouvait que c'était le parent pauvre " (CG) ; " Avant les Feuilles de géographie était le néant. [rires][...]Non mais sans rire, avant les feuilles de géographie c'était le néant pédagogique à l'Université. [...] C'est pas parce que t'as des mômes qui ont 18ans et demi que tu vas pas te préoccuper de questions pédagogiques, alors qu'on s'en préoccupe quand ils ont 17 ans et demi! Donc, le premier geste militant de l'assoc' et de la revue c'était ça!" (GZ).

\section{"Casser les chapelles, virer les mandarins " (RLG)}

Un certain nombre d'ambitions peuvent être liées à une volonté de rupture par rapport à l'organisation même des rapports de force et des clivages à l'intérieur de la discipline,ainsi que la volonté de ne pas être captifs de ceux créés auparavant : " nos aînés [...] avaient eu leurs querelles, leurs guerres de tranchées, avec la géopolitique de Lacoste, avec les positivistes quantitatifs, les culturalistes... En gros l'idée c'est qu'on n'avait pas envie $d^{\prime}$ 'hériter des querelles. [...] On était prêt à se quereller entre nous mais [rires] on ne voulait pas hériter des querelles précédentes. On voulait pouvoir choisir les nôtres »(CG); "Avec après aussi dans les objectifs des trucs 'on va casser les chapelles' " (GZ), "virer les mandarins » (RLG). 
Pour ces allocataires moniteurs,l'un des objectifs de Feuilles de Géographie était aussi orienté vers la remise en cause de la hiérarchie traditionnelle entre les enseignements "nobles", thématiques et/ou théoriques face aux outils.L'ambition était pour eux de démontrer la nécessité d'une réflexion sur la pédagogie des outils: "Le premier tabou c'était quand même l'histoire qu'il y avait les outils et les méthodes, untruc qui était, voilà :c'était le prolétariat. Et puis il y avait les enseignements nobles, la géomorphologie... Donc nous déjà on estimaitqu'il n'y avait pas de hiérarchie entre les enseignements et qu'il fallait les croiser » (CG). II s'agissait également de revaloriser les Travaux Dirigés (TD) face au traditionnel Cours Magistral (CM) :« Une inspiration supplémentaire, qui était le fait de structurer davantage, et de faire connaître davantage les TD qui ne devaient pas être seulement des faire-valoir des cours magistraux. [...] C'est-à-dire qu'il y avait la séquence du cours magistral qui abordait la thématique, que les étudiants devaient savoir, et puis après... On la mettait comme on pouvait en musique, sans que cela soit vraiment satisfaisant. Et donc l'idée, c'était que, au même titre qu'il y avait une progression dans la réflexion du cours magistral, il devait y avoir aussi une progression en termes de TD, réflexion, capacité à s'approprier un corpus de documents, capacité à traiter l'information, etc. [...] L'exigence de l'équipe pédagogique ou de l'animateur de l'équipe pédagogique, c'était de nous dire : vous récupérez des matériaux pédagogiques à la fin de l'année, il faut que vous les enrichissiez. Et donc à la fin de l'année, il faut que vous proposiez un nouveau TD, une nouvelle séquence etc., qui à son tour sera transmise au fond commun et qui sera transmis aux personnes qui vont être intégrées l'année suivante " (MB). Dans cette logique, les membres de Feuilles de Géographie ont contribué à la promotion de formules hybrides entre CM et TD, tels que les cours intégrés dans les maquettes alors en place à l'Université Paris $7^{8}$.

\section{Autogestion et pragmatisme}

A ses débuts, la revue s'oriente assez rapidement vers un mode de gestion coopératif, tout en assumant une exigence forte de qualité dans le contenu des Feuilles, relues puis testées en situation de cours.

«CG : Bah disons dans les deux courants du XIX siècle, on était plutôt dans I'syndicalisme autogestionnaire quoi [rires], bon, et non pas révolutionnaire, on était dans l'autogestion.

MB : Mais avec une dimension, on était quand même dans des équipes où il y avait une grosse exigence de qualité, on pouvait se gaufrer sur une séance de $T D$, mais il était hors de question de se gaufrer sur un semestre. Donc y'avait le filet de sécurité des collègues qui avaient déjà une ou deux années d'bouteille dans l'équipe pédagogique. Mais on avait aussi une exigence de production de qualité qui était à la hauteur, qui était susceptible d'être repris ".

LesFeuilles de géographiesemblent offrir une réponse pragmatique à des besoins et des problèmes concrets, tout en refusant l'appartenance à une école de pensée didactique :«

\footnotetext{
${ }^{8}$ Notons qu'aucune référence $n$ 'est faite aux innovations pédagogiques développées au centre expérimental de Vincennes à partir des années 1970, comme les cours intégrés CM-TD encore en vigueur à Paris 8 Vincennes Saint-Denis.
} 
comme la revue La Classe", on n'était pas rattaché à une école didactique " (CG) "l'idée ce n'était pas de dire j'oriente mon TD de cette manière-là, je présente comme ci comme ça. C'est plutôt de dire, voilà, j'ai cette question à traiter, voilà les documents que je vous propose de mobiliser, et voilà les points saillants de ma démonstration » (MB).

Tout aussi concrètement, certains ont pu être attirés par la revue pour approfondir leurs premières expériences pédagogiques : "moi c'était mon bizutage à l'enseignement " (RLG), "Ce qui m'attirait, c'est le côté collectif des cours, moi je ne l'avais pas du tout rencontré en tant que monitrice. Parce que ça dépend quel type de cours tu fais. Ils étaient dans la bibliothèque de la rue du Four ${ }^{10}$ tu sais, et moi j'étais hyper curieuse de ça, de voir comment les TD étaient construits tout ça, parce que moi en tant que monitrice, je n'ai jamais eu de formation. Il y avait le fameux $C_{E S S^{11}}$, mais il ne nous apprenait pas à faire des cours. On faisait du théâtre, c'était sympa, mais bon. [...] C'est vraiment ça qui m'a intéressée: formuler des objectifs, voir un peu la quantité de documents que tu pouvais apporter dans un cours » (AR).

Le cœur du système des Feuilles de géographiereposait sur l'investissement conséquent d'un réseau actif et d'un noyau dur.La quantité de travail était importante, surtout pour ceux qui étaient chargés des travaux les plus fastidieux de mise en page, impression, collage et expédition des numéros imprimés, voire parfois du transport des Feuilles jusqu'à Saint-Dié lors duFestival de géographie annuel.La revue ne pouvait pas fonctionner sans compter sur la participation active de ses lecteurs, également membres de l'association et alimentant les nouveaux numéros. La structure associative a d'ailleurs servi de support à la diffusion dans certaines universitésen nommant temporairement des responsables éditoriaux pour la publication de numéros spécifiques (exemple du numéro Grand Ouest,coordonné par Vincent Veschambre).

\section{La reconnaissance institutionnelle}

Le succès de la revue a dépassé les attentes initiales, aboutissant à une certaine reconnaissance institutionnelle : " un critère de succès qu'on s'était donné, je ne sais pas si on l'a atteint, c'était de se dire, en gros, on aura réussi l'objectif de la revue le jour où, soit au CNU, soit dans un comité de recrutement, on dira il a fait une Feuille de géographie donc... » (CG). Ce qui arriva assez rapidement pour certains une fois la période de recrutement arrivée : "c'est aussi parce que j'avais trois feuilles de chou que mon dossier était considéré comme bon dossier à l'arrivée »(GZ).

Ensuite, un certain nombre de membres de l'équipe ont étérecrutés comme titulaire, ce qui pour eux signifiait ancrer leurs réflexions pédagogiques dans l'institution : "Je pense aussi qu'on est arrivé à un moment, je pense aux recrutements, etc., où ces questions pédagogiques sont devenues importantes parce que l'environnement a changé

\footnotetext{
${ }^{9}$ Cette revue, aujourd'hui en ligne (http://www.laclasse.fr/) est un espace de partage de séquences de cours entre enseignants du primaire. Elle a inspiré la forme coopérative des Feuilles de géographie.

${ }^{10}$ Bibliothèque de l'équipe P.A.R.I.S., U.M.R. Géographie-cités, situé au 13 rue du Four dans le sixième arrondissement de Paris.

${ }^{11}$ Centre d'Initiation à l'Enseignement Supérieur.
} 
considérablement, notamment au ministère où avec le passage au LMD il y a eu cette pression institutionnelle, de transformation de l'institution. „(RLG).

Ainsi, l'iconoclastie des "casseurs de chapelle " ne rencontre pas une si vive opposition: " on les a [au sujet des réticences à la revue] assez peu senties, il faut être honnête. [...] parce qu'on était aussi pris un peu dans notre bulle, dans notre truc, etc., et on avançait " (MB). Et la dynamique des Feuilles de géographie se poursuit, jusqu'auralentissement de l'activité éditoriale au milieu des années 2000.

\section{« On a senti l'obsolescence venir extrêmement rapidement » (RLG)}

Au cours de quinze ans d'activité, Feuilles de géographie a dû faire face à un certain nombre de mutations de l'enseignement supérieur et de la recherche, questionnant la pertinence actuelle de son fonctionnement.

\section{La logique contributive face aux évolutions de l'enseignement supérieur}

Le recrutement progressif de certains membres historiques actifs pose la question du renouvellement générationnel :" parce que les gens sont recrutés et qu'on est pris par nos charges par ailleurs [...]. On est pris par des charges, par des animations d'équipes [...]. Enfin c'est un peu le même genre de boulot pédagogiquement, mais on est aspiré par d'autres tâches tout simplement " (AR); "dans l'absolu rien ne t'empêche de continuer, mais c'est le temps. Et puis au bout d'un moment, on avait l'impression que ça ne se renouvelait pas non plus et puis au niveau de l'énergie, tu n'es plus sur l'innovation, le départ, etc. " (SB).La lourdeur organisationnelle et logistique de l'époque pesant sur un petit groupe de coordinateurs $^{12}$ se ressentait sur la dynamique de publication des Feuilles: effectivement cette lourdeur qui contribuait à l'absence de périodicité claire ${ }^{13}$.[...]On faisait comme on pouvait en fait " (GZ). De plus, l'investissement temporel apparaît d'autant plus coûteux que ce travail éditorial reste peu valorisé par rapport aux revues scientifiques. Surtout, la dynamique de travail collectif peine à se renouveler compte tenu des différents niveaux d'intégration des groupes d'une génération à l'autre: "Tu sais, il n'y a pas forcément beaucoup de liens entre les générations, entre les générations de doctorants. On était très soudés [...].C'est des configurations à un moment donné, c'est très conjoncturel »(AR).

Mais ce déclin tiendrait aussi, selon les anciens membres, aux évolutions structurelles de l'enseignement supérieur et des attentes vis-à-vis des publications : "Donc, comment tu fais pour demander à quelqu'un un retour qui est l'engagement militant de 'moi aussi je vais fournir aux autres, quelque chose qui va alimenter la feuille et qui va nourrir du monde'. Et

\footnotetext{
12 Selon les comptes rendus des assemblées générales de l'association entre 2003 et 2005, ces réunions comptaient une dizaine de personnes, tandis que l'activité quotidienne reposait plutôt sur moins de cinq membres.

${ }^{13}$ De 1994 à 2015, plus d'une cinquantaine de Feuilles ont été publiées, avec une moyenne d'environ six Feuilles pour les premières années, puis quatre Feuilles en moyenne par année, avec des interruptions d'une ou deux années.
} 
donc si tu veux, la logique, pour moi, ce que je voyais disparaître, du fait de ce contexte vraiment, c'est la logique de banque alimentaire. C'est bien de distribuer aux pauvres les paquets de riz, mais si au bout d'un moment plus personne ne ramène de paquet de riz... La logique est perdue. Et moi, c'est ça que j'ai vu au milieu des années 2000 [...] " (GZ). Pris dans une logique individualiste et consumériste, le modèle coopératifde la revueaurait perdude son sens: "Au fond, c'est pas ça qui fonctionne, parce que, c'est alimenté par une seule source et ça s'épuise très très vite. C'est-à-dire que si t'as pas de contre partie... Si tu entends préparer un TD et que t'as pas la contrepartie de, je vais pas dans le lot en recevoir un ou deux, l'idée de fonctionner en coopérative, comme le font les collègues qui doivent préparer des nouvelles questions tous les ans... Parce qu'en fait un doctorant il est dans la même situation. Il change de poste au bout d'un an... Enfin, il a trois ans dans un endroit, et au bout de trois ans, bam ! [...] c'est là qu'appartenir à un réseau, à une coopérative, en se disant : 'eh bien voilà, je vais me retrouver à enseigner ailleurs et je vais peut-être vouloir échanger avec les gens " (RLG); "le problème est lié à la notion de passivité quand t'es consommateur d'une revue » (GZ).

\section{Le tournant des années 2000 : nouvelles technologies, nouveaux rapports à l'enseignement et aux étudiants?}

Plus généralement,les anciens membres constatent une transformation des supports, des méthodes de diffusion, mais aussi de la manière d'enseigner et de la relation aux étudiants elles-mêmes, transformation qu'ils situent au tournant des années 2000. Le tournant numérique du début de cette période pose concrètement le problème de la diffusion des Feuilles et modifie durablement les pratiques d'enseignement.

De retour d'un terrain aux États-Unis, Renaud Le Goix prend l'initiative de changer de format et de passer à une version numérique des Feuilles de géographie: "Moi j'étais élevé à l'âge de l'internet dynamique, je savais coder en .php, je savais coder en .html, donc je me suis dit : 'allez, il faut y aller'. Et j'ai pris mon mois d'août où je me suis dit : 'je vais coder. Je vais faire mon truc en .php. Hop. Une base de données en .sql, on pourra alimenter le truc, faire des liens, voilà. Et une page dynamique, où on pourra évoluer'.[...]Et, en même temps, c'était super intéressant et extrêmement frustrant parce que j'ai senti que le modèle s'effondrait. [...] J'ai senti qu'en faisant ça on annulait le modèle. En fait le modèle il était annulé déjà. Au fond, c'est la révolution de l'accès à l'information qui avait changé. " (RLG). En effet, l'accès illimité à l'information via Internet rend moins pertinente la publication de cours formalisés: "T'as trois documents de TD. Tu survis, tu survis $2 h$. Le besoin même d'un support construit, encadré, devenait un peu plus évanescent.[...]On a senti l'obsolescence venir extrêmement rapidement » (RLG).

L'un des changements majeurs de la période concerne bien "l'accès aux sources [...] fantastiquement facile, où on peut travailler sur des choses en ligne directe, en même temps, avec les étudiants, pour qu'ils soient en apprentissage.[...] Comme ils ont tous un ordinateur devant eux, de jouer et de théâtraliser sur le fait de leur faire vérifier des choses "(RLG). Ces changements, au-delà du rythme et de la forme du cours, bouleversent également le contenu : 
« GZ : "Sachant que je sers pas à diffuser de la connaissance. Il y a plus de connaissance dans Internet et Wikipédia que j'en ai dans mon cerveau. Et que je ne pourrais jamais transmettre en 26 heures de TD semestre. [...] Donc, spontanément, au milieu des années 2000, moi j'ai pris le tournant méthodo.

RLG : Voilà. C'est ça, voilà. Et donc t'introduis des changements, des ruptures, etc. Donc tu penses ton cours autrement, y compris en termes de rythme de cours $"$.

\section{LesFeuilles de Géo face à la concurrence ${ }^{14}$}

Malgré différentes tentatives d'adaptation à ces évolutions, notamment éditoriales (éditions de numéros hors-série, publication de progression de cours, Travaux Dirigés sur la pratique d'outils appliqués à des exemples thématiques) et techniques (édition de CD'roms par exemple), Feuilles de Géo doit faire face à une diversification de l'offre à vocation pédagogique: "Géoconfluences, quand même ! C'est arrivé à ce moment-là. Avec la puissance de l'ENS de Lyon. La puissance du $C N D P^{15}$ et donc des institutions derrière, surtout du CNDP. Mais le moteur scientifique de la publication c'était l'ENS. Et mine de rien, ça a siphonné le réseau des normaliens. C'est-à-dire que Géoconfluences est devenu le vecteur de publications des jeunes thèses innovantes pour une publication en ligne. Pour l'article scientifique rang $A$, tu as Cybergéo. Tu vas dans d'autres revues qui ont explosé depuis. Pour la publication orientée pédago, de valorisation de la thèse vers un usage dans l'enseignement, tu vas à Géoconfluences, qui a, euh, su innover avec des moyens techniques professionnels. Parce que derrière il y avait des moyens professionnels. [...]Et une vraie politique éditoriale. Et donc, quelque part, quand Géoconfluences a émergé, littéralement, on a vu que les réseaux de publication commençaient à se transformer 》 (RLG).

Cette concurrence interroge le format et les modalités de publication: les étapes successives, faisant passer lesFeuilles de géographied'une revue papier accessible par adhésion payante à l'association, à une revue en ligne payante, puis gratuite par inscription, pour enfin devenir une revue en ligne gratuite et libre d'accès semble suivre la trajectoire de modes de consommations de moins en moins contraignants. De ce fait, la question de la participation des consommateursde la revue pour la faire vivre et maintenir la dimension coopérative est plus que jamais d'actualité, si l'on considère quepartager des supports de cours et discuter de pédagogie à l'Université en géographie a toujours un sens.

\footnotetext{
${ }^{14}$ Le terme de concurrence est utilisé par les anciens membres ; il est en même temps révélateur des logiques institutionnelles universitaires.

${ }^{15}$ Centre National de Documentation Pédagogique (situé au sein de la bibliothèque Diderot, adossée au campus de l'ENS de Lyon dans le quartier de Gerland, Lyon).
} 


\section{Conclusion: "Parce que la pédagogie dans le supérieur, le problème il est pas réglé ॥ (GZ)}

Comment faire cours à l'heure du numérique : " C'est moi la prof, c'est pas Wikipédia ! " (GZ)

À partir de ces retours d'expériences, il semble que la question de la pédagogie soit toujours d'actualité, même si elle est loin de se poser de la même manière qu'il y a dix ou quinze ans. "J'en suis revenu. Parmi les évolutions pédagogiques, après les avoir subies, tout ça, voilà, formé à l'ancienne école et venu s'adapter au truc, j'me rends compte maintenant que je suis revenu à des choses très très hybrides et que j'évolue dans ma manière d'enseigner. J'étais un fervent défenseur des powerpoints, des nouvelles technologies en cours, des machins, etc., et maintenant je me retrouve à dire, quand je fais un cours d'amphi en première année : ils vont voir ce que c'est qu'un cours d'amphi, structuré, machin, etc., la rhétorique, la transition, le machin, mais avec des pauses avec une vidéo, un machin, pour... Parce qu'il faut capter le public autrement »(RLG).Plus largement, une réflexion globale et collective s'impose sur l'intégration - ou non - des outils numériques dans les pratiques pédagogiques. Comment créer des rythmes et des dynamiques de cours ne s'appuyant pas uniquement sur la performance visuelle de supports numériques? Se réserver le temps pour un débat permettant le dialogue entre les générations ayant connu cette transition technologique et celles dont elles sont le produit semble aujourd'hui plus que nécessaire.

\section{Les impensés de la pédagogie à l'Universitéetde nouveaux chantiers pour la revue}

Un certain nombre d'angles morts de la revue pourrait être comblés, notamment autour des thématiques liées aux questions d'aménagement, aux " pays en voie de développement ", à la géographie régionale ou encore à la géographie physique ou environnementale. Évidemment, une actualisation des Feuilles est nécessaire pour qu'elles correspondent à la diversité des géographies contemporaines et à leurs relations avec d'autres champs disciplinaires (sociologie, arts, écologie, etc.).Une plus grande ouverture vers d'autres pays pourrait également être bénéfique à la diffusion des manières de faire. Des expériences menées à l'étrangeravaient en effet contribué pour certains membres à changer de perspective sur leurspratiques (exemple d'une Feuille sur la dissertation, discutée à partir d'exemples belges).

De plus, une réflexion de fond sur l'articulation des enseignements au sein des formations universitaires en géographie reste à construire : qu'enseigne-t-on en géographie dans les différentes universités ? Quelles réalités recoupent un même intitulé ? Et réciproquement, quelles nuances et visions de la géographie peuvent s'appréhender dans la diversité des intitulés et des maquettes ? LesFeuilles de géographiese sont concentrées sur les enjeux pédagogiques des formations de Licence, qu'en est-il des enjeux pédagogiques dans les deuxièmes et troisièmes cycles ? : Q Qu'est-ce que ça veut dire enseigner à chercher ? [...] Entre la Licence et le Master, nous, on a essuyé les plâtres et on a épongé une grosse partie de la question en licence. Il faut toujours renouveler, parce que, plein de choses ont 
changé » (GZ) ; "La question du parcours pédagogique en thèse. C'est un impensé de notre discipline. Totalement » (RLG).

Plus largement, un travail sur la pédagogie même, à différentes échelles (au sein du groupe classe, des équipes, des départements) serait nécessaire. Si on entend la pédagogie comme l'articulation des modalités de transmission de savoirs et de savoir-faire, plusieurs axes de réflexion nous semblent intéressants à approfondir: l'intégration de la question des inégalités sociales dans les pratiques pédagogiques, la question de la notation et de l'évaluation des étudiants, l'enjeu de la situation pédagogique dans ses différentes dimensions (matérielle, sensible, relationnelle, etc.), la variation des rythmes et supports d'enseignement (outil informatique, construction des séquences), l'évolution et les sens du métier (et de la formation au métier) d'enseignant-chercheur.

Investir et réinvestir des espaces de réflexion, d'échanges et de production collective revêt une importance accrue dans un contexte d'isolement de certains enseignants aux statuts toujours plus variés, de mise en concurrence des apprentis enseignants-chercheurs, et de pénurie et de précarisation dans l'enseignement supérieur.Ces éléments paraissentd'autant plus importants que la demande sociale par rapport à l'Universitésemble évoluer: articulation entre enseignements thématiques et enseignements d'outils et de méthodes, exigence accrue de professionnalisation, nouvelles attentes et représentations de I'Université dans un contexte de massification des effectifs, de précarisation de l'accès à l'emploi, et de transformation du système de l'Enseignement Supérieur et de la Recherche (gouvernance, évaluation, financement, etc.).Autant d'évolutions qui interrogent les missions et les fonctions de l'Université. Réfléchir aux méthodes pédagogiques et renforcer la dimension collective de l'enseignement au sein des universités et entre elles paraît donc crucial tant pour les contenus que pour revaloriser cette activité centrale de notre métier. 